\title{
Intrinsic relation between ground-state fidelity and the characterization of a quantum phase transition
}

\author{
Shu Chen, Li Wang, Yajiang Hao and Yupeng Wang \\ Institute of Physics, Chinese Academy of Sciences, Beijing 100080, China
}

(Dated: October 31, 2018)

\begin{abstract}
The notion of fidelity in quantum information science has been recently applied to analyze quantum phase transitions from the viewpoint of the ground-state (GS) overlap for various many-body systems. In this work, we unveil the intrinsic relation between the GS fidelity and the derivatives of GS energy and find that they play equivalent role in identifying the quantum phase transition. The general connection between the two approaches enables us to understand the different singularity and scaling behaviors of fidelity exhibited in various systems on general grounds. Our general conclusions are illustrated via several quantum spin models which exhibit different kinds of QPTs.
\end{abstract}

PACS numbers: 03.65.Ud, 64.60.-i, 05.70.Jk, 75.10.-b

\section{INTRODUCTION}

Quantum phase transitions (QPTs) that happened at the zero temperature is purely a phenomenon of ground state (GS) transition driven by external parameters. Traditionally, QPTs are described in terms of order parameter and symmetry breaking within the Landau-Ginzburg paradigm which have been extensively studied in condensed matter physics [1]. In recent years, QPT has also attracted a lot of attention in quantum-information science [2, 3], in which one of the research focus is the role of quantum entanglement in characterizing QPTs 2, 3, 4, 5, 6]. More recently, another concept in quantum information science, i.e., the fidelity has been put forward to identify QPTs from the perspective of the GS wave functions [7, 8]. The GS fidelity is defined as the overlap between two ground states with only slightly different values of the external parameters [8] and thus is a pure geometrical quantity. Since no a priori knowledge of the order parameter is needed, the fidelity might be a potential universal criteria for characterizing the QPTs 8, 9, 10, 11, 12, 13, 14, 15]. An increasing interest has been drawn in the role of GS fidelity in detecting QPTs for various many-body systems $9,10,11,12,13,14,15]$, since Zanardi and Paunkovic first exploited it to identify QPTs in the XY spin chain [8] where the fidelity shows a narrow drop at the transition point. Remarkably, the success of fidelity analysis in dealing with the Bose-Hubbard model [9] and spin systems [14, 15] implies that it may have practical relevance even for more complicate strongly interacting systems where no a simple description is possible. The relation between the fidelity and Berry phase [16] has also been unveiled in terms of Riemannian metric tensors [17].

Generally one may expect that the GS fidelity shows an abrupt drop in the vicinity of the QPT point of the system as a consequence of the dramatic change of the structure of the GS. This is true for the first-order QPTs caused by a level crossing of GSs due to the GS wavefunctions at the different sides of the level-crossing point are almost orthogonal (orthogonal at the level-crossing point). However, this is neither an obvious nor a general conclusion for a continuous QPT where no GS level crossing occurs and the GS evolves "adiabatically" in the parameter space [11, 14]. Conventionally, QPTs are characterized by singularities of the ground state energy: first-order QPTs are characterized by discontinuities in the first derivative of the energy, whereas second-order (nth-order) QPTs are characterized by discontinuities in the second ( $n$ th) derivative of the energy. An important question is whether the singularity of GS energy is intrinsically related to the GS overlap? Answering this question is no doubt significant for a deeper understanding of QPTs, and the validity and limitation of fidelity as a measure of QPTs.

So far the studies of fidelity as a measure of QPTs are based on the analysis of particular many-body models [8, 9]. The general connection between GS fidelity and QPTs is not yet well established. In this work, we shall discuss, in a general framework, how the GS fidelity can be related to a QPT characterized by non-analyticities of the GS energy. Our result shows that the singularity and scaling behaviors of the GS fidelity (or it derivatives) are directly related to its correspondences of the derivative of GS energy.

Our paper is organized as follows. In section II, we reveal the general relation between the fidelity susceptibility and the 2nd order derivative of the GS energy. The subsequent sections are devoted to two examples which exhibit the 2nd order and Kosterlitz-Thouless (KT) QPTs, respectively. A summary is given in the last section.

\section{FIDELITY AND QUANTUM PHASE TRANSITION}

The general Hamiltonian of a quantum many-body system undergoing QPTs reads

$$
H(\lambda)=H_{0}+\lambda H_{1},
$$

where $H_{1}$ is supposed to be the driving term with $\lambda$ the control parameter. In terms of the eigenstates $\left|\Psi_{n}(\lambda)\right\rangle$ of 
$H(\lambda)$, the Hamiltonian can be reformulated as $H(\lambda)=$ $\sum_{0}^{\mathcal{N}-1} E_{n}(\lambda)\left|\Psi_{n}(\lambda)\right\rangle\left\langle\Psi_{n}(\lambda)\right|$, where $\mathcal{N}$ is dimensions of the Hilbert space. The GS fidelity is defined as the overlap between $\left|\Psi_{0}(\lambda)\right\rangle$ and $\left|\Psi_{0}(\lambda+\delta)\right\rangle$, i.e.

$$
F(\lambda, \delta)=\left|\left\langle\Psi_{0}(\lambda) \mid \Psi_{0}(\lambda+\delta)\right\rangle\right|,
$$

where $\Psi_{0}(\lambda)$ is the GS wavefunction corresponding to the parameter $\lambda$ and $\delta$ is a small quantity [8]. It is obvious that the fidelity is dependent of $\delta$. The rate of change of fidelity is given by the second derivative of fidelity [8] or fidelity susceptibility (FS) [1]

$$
S(\lambda)=\left.\partial_{\delta}^{2} F(\lambda, \delta)\right|_{\delta=0} \simeq 2 \lim _{\delta \rightarrow 0} \frac{1-F(\lambda, \delta)}{\delta^{2}},
$$

which is $\delta$ independent and sometimes a more effective quantity to detect the QPT.

First we discuss the first order QPT (1QPT) which is induced by GS level crossing. We assume $E_{0}(\lambda)<E_{1}(\lambda)$ for $\lambda<\lambda_{c}$ with $\lambda_{c}$ being the crossing point where the GS energy level $E_{0}$ crosses over the first excited level $E_{1}$, therefore we must have $E_{1}(\lambda)<E_{0}(\lambda)$ for $\lambda>\lambda_{c}$. Explicitly, for the 1QPT, the GS energy is defined as

$$
E_{g}(\lambda)= \begin{cases}E_{0}(\lambda) & \lambda<\lambda_{c} \\ E_{1}(\lambda) & \lambda>\lambda_{c}\end{cases}
$$

The derivative of the GS energy is defined as

$$
\frac{\partial E_{g}(\lambda)}{\partial \lambda}= \begin{cases}\lim _{\lambda \rightarrow \lambda_{c}-0} \frac{E_{0}(\lambda)-E_{0}\left(\lambda_{c}\right)}{\lambda-\lambda_{c}}=\frac{\partial E_{0}(\lambda)}{\partial \lambda} & \lambda<\lambda_{c}, \\ \lim _{\lambda \rightarrow \lambda_{c}+0} \frac{E_{1}(\lambda)-E_{1}\left(\lambda_{c}\right)}{\lambda-\lambda_{c}}=\frac{\partial E_{1}(\lambda)}{\partial \lambda} & \lambda>\lambda_{c} .\end{cases}
$$

In general, $\frac{\partial}{\partial \lambda} E_{0}(\lambda)$ is not equal to $\frac{\partial}{\partial \lambda} E_{1}(\lambda)$, therefore the first derivative of GS energy for a 1QPT is not continuous at the transition point. Accordingly, the GS fidelity at the transition point is defined as $F\left(\lambda_{c}, \delta\right)=\left|\left\langle\Psi_{g}\left(\lambda_{c}-\delta / 2\right) \mid \Psi_{g}\left(\lambda_{c}+\delta / 2\right)\right\rangle\right|=$ $\left|\left\langle\Psi_{0}\left(\lambda_{c}-\delta / 2\right) \mid \Psi_{1}\left(\lambda_{c}+\delta / 2\right)\right\rangle\right|$, therefore we have $\lim _{\delta \rightarrow 0} F\left(\lambda_{c}, \delta\right)=0$ which means that there appears a sharp drop in the transition point. It is straightforward that the sudden drop in the transition point shares the same physical origin with the discontinuity of the first derivative of GS energy.

Next we consider the continuous phase transition for which the GS of the Hamiltonian is nondegenerate for a finite system. For the case of $2 n d$ order QPT, there is no level crossing for the GS energy, therefore we can always represent $E_{g}(\lambda)=E_{0}(\lambda)$. The first derivative of the GS energy is given by $\frac{\partial}{\partial \lambda} E_{0}(\lambda)=\left\langle\Psi_{0}(\lambda)\left|H_{1}\right| \Psi_{0}(\lambda)\right\rangle$, which is noting else but the Hellmann-Feynman theorem. It is straightforward to get the second derivative of GS energy $\frac{\partial^{2}}{\partial \lambda^{2}} E_{0}(\lambda)=\left\langle\Psi_{0}(\lambda)\left|H_{1}\right| \partial_{\lambda} \Psi_{0}(\lambda)\right\rangle+$ h.c. . Inserting the identity operator $\sum_{n=0}^{\mathcal{N}-1}\left|\Psi_{n}(\lambda)\right\rangle\left\langle\Psi_{n}(\lambda)\right|=1$ between $H_{1}$ and $\left|\partial_{\lambda} \Psi_{0}(\lambda)\right\rangle$, we then get $\frac{\partial^{2}}{\partial \lambda^{2}} E_{0}(\lambda)=$ $\sum_{n=1}^{\mathcal{N}-1} H_{0 n}^{1}\left\langle\Psi_{n}(\lambda) \mid \partial_{\lambda} \Psi_{0}(\lambda)\right\rangle+$ h.c. with $H_{0 n}^{1}=$ $\left\langle\Psi_{0}(\lambda)\left|H_{1}\right| \Psi_{n}(\lambda)\right\rangle$. We note that the term of $n=0$ is not included in the summation because it gives zero due to $\left\langle\partial_{\lambda} \Psi_{0}(\lambda) \mid \Psi_{0}(\lambda)\right\rangle+\left\langle\Psi_{0}(\lambda) \mid \partial_{\lambda} \Psi_{0}(\lambda)\right\rangle=0$. Differentiating the eigenequation $H\left|\Psi_{0}(\lambda)\right\rangle=E_{0}\left|\Psi_{0}(\lambda)\right\rangle$ with respect to $\lambda$ and taking the inner product with $\Psi_{n}(\lambda)$ yields $\left\langle\Psi_{n}(\lambda)\left|\partial_{\lambda} H\right| \Psi_{0}(\lambda)\right\rangle+\left\langle\Psi_{n}(\lambda)|H| \partial_{\lambda} \Psi_{0}(\lambda)\right\rangle=$ $\partial_{\lambda} E_{0} \delta_{n 0}+E_{0}\left\langle\Psi_{n}(\lambda) \mid \partial_{\lambda} \Psi_{0}(\lambda)\right\rangle$. Exploiting the hermiticity of $H$ to write $\left\langle\Psi_{n}(\lambda)|H| \partial_{\lambda} \Psi_{0}(\lambda)\right\rangle=$ $E_{n}\left\langle\Psi_{n}(\lambda) \mid \partial_{\lambda} \Psi_{0}(\lambda)\right\rangle$, it follows that for $n \neq 0$, $\left\langle\Psi_{n}(\lambda) \mid \partial_{\lambda} \Psi_{0}(\lambda)\right\rangle=\left\langle\Psi_{n}(\lambda)\left|\partial_{\lambda} H\right| \Psi_{0}(\lambda)\right\rangle /\left(E_{0}-E_{n}\right)$. Therefore, we have

$$
\frac{\partial^{2}}{\partial \lambda^{2}} E_{0}(\lambda)=\sum_{n \neq 0} \frac{2\left|\left\langle\Psi_{n}(\lambda)\left|H_{1}\right| \Psi_{0}(\lambda)\right\rangle\right|^{2}}{E_{0}(\lambda)-E_{n}(\lambda)} .
$$

To see the relation of FS with the second derivative of GS energy, we rederive the expression of FS [11, 17] by expanding the wavefunction $\left|\Psi_{0}(\lambda+\delta)\right\rangle$ in the basis of eigenstates corresponding to the parameter $\lambda$, to the first order, which leads to

$$
\left|\Psi_{0}(\lambda+\delta)\right\rangle=c\left(\left|\Psi_{0}(\lambda)\right\rangle+\delta \sum_{n \neq 0} \frac{H_{n 0}^{1}(\lambda)\left|\Psi_{n}(\lambda)\right\rangle}{E_{0}(\lambda)-E_{n}(\lambda)}\right),
$$

where $c=\sqrt{1+\delta^{2} \sum_{n \neq 0}\left|H_{n 0}^{1}(\lambda)\right|^{2} /\left[E_{0}(\lambda)-E_{n}(\lambda)\right]^{2}}$ is the normalization constant. It follows directly $F(\lambda, \delta)=$ c. Substituting it back into Eq. (3), we get

$$
S(\lambda)=\sum_{n \neq 0} \frac{\left|\left\langle\Psi_{n}(\lambda)\left|H_{1}\right| \Psi_{0}(\lambda)\right\rangle\right|^{2}}{\left[E_{0}(\lambda)-E_{n}(\lambda)\right]^{2}} .
$$

Comparing Eqs. (5) and (4), we find that for both of them the singularities come from the vanishing energy gap in the thermodynamic limit. However, a divergence does not necessarily occur in the critical point, for example, for the KT QPT as we shall discuss later, no singularity arises even the energy gap tends to zero in the thermodynamic limit as matrix elements $H_{n 0}^{1}$ may also vanish simultaneously. Despite the apparent similarity between Eqs. (5) and (41), we note that fidelity susceptibility cannot be expressed by the GS energy 2nd derivatives alone [18]. Nevertheless, the different power in the denominators in Eqs. (5) and (41) shows that fidelity susceptibility might be a more sensitive tool to detect critical points because it can be more singular than the second derivative of GS energy. Therefore, for the case where the GS energy 2nd derivative is divergent at the critical point, it is no doubt that the fidelity susceptibility is divergent too. However, for the case where the second derivative of GS energy is not divergent, (for example the 3rd-order QPTs and the KT phase transition), it is hard to judge whether the fidelity susceptibility becomes singular or not at these critical points just from the relation between Eqs. (5) and (4).

\section{TRANSVERSE FIELD ISING MODEL}

To understand the equivalence of the two approaches in characterizing the quantum criticality, we first apply 
them to study a specific example, say, the transverse field Ising model with the Hamiltonian given by

$$
H(\lambda)=-\sum_{i=1}^{L}\left(\hat{\sigma}_{i}^{x} \hat{\sigma}_{i+1}^{x}+\lambda \hat{\sigma}_{i}^{z}\right)
$$

where $\lambda$ represents external magnetic field along the $z$ axis and the periodic boundary is assumed. Following the standard procedure [1, 19] , the model can be diagonalized by applying Jordan-Wigner transformation which maps the spin model to a fermion model and then using the Bogoliubov transformation $c_{k}=b_{k} \cos \frac{\theta_{k}}{2}-i b_{-k}^{\dagger} \sin \frac{\theta_{k}}{2}$. The result is given by $H=\sum_{k} \omega_{k}\left(c_{k}^{\dagger} c_{k}-1\right)$ with $\omega_{k}=$ $\sqrt{\sin ^{2} \frac{2 \pi k}{L}+\left(\lambda-\cos \frac{2 \pi k}{L}\right)^{2}}$, where the summation is over $k=-M, \cdots, M$ with the assumption of $L=2 M+1$. The ground state of $H$ is the vacuum $|g\rangle$ of $c_{k}$ given by $|g\rangle=\prod_{k=1}^{M}\left(\cos \frac{\theta_{k}}{2}|0\rangle_{k}|0\rangle_{-k}-i \sin \frac{\theta_{k}}{2}|1\rangle_{k}|1\rangle_{-k}\right)$, with the ground state energy $E_{0}(\lambda)=-\sum_{k} \omega_{k}$, where $|0\rangle_{k}$ and $|1\rangle_{k}$ are, respectively, the vacuum and single excitation of the $k$ th mode $b_{k}$ and $\theta_{k}$ is defined by $\sin \theta_{k}=\sin \frac{2 \pi k}{L} / \omega_{k}$. It follows that the fidelity is given by $F(\lambda, \delta)=\prod_{k=1}^{M} \cos \frac{\theta_{k}-\widetilde{\theta}_{k}}{2}$ with $\widetilde{\theta}_{k}=\theta_{k}(\lambda+\delta)$ [8]. After some very simple algebras, one get $S(\lambda)=$ $2 \frac{1-F(\lambda, \delta)}{\delta^{2}}=\frac{1}{4} \sum_{k=1}^{M}\left(\frac{\partial \theta_{k}}{\partial \lambda}\right)^{2}$. In the thermodynamic limit with $L \rightarrow \infty$, the summation can be replaced by the integral and thus we get

$$
\frac{\partial^{2}}{\partial \lambda^{2}} e_{0}(\lambda)=-\frac{1}{2 \pi} \int_{-\pi}^{\pi} \frac{\sin ^{2} \varphi}{\left[\sin ^{2} \varphi+(\lambda-\cos \varphi)^{2}\right]^{3 / 2}} d \varphi
$$

and

$$
s(\lambda)=\frac{1}{16 \pi} \int_{-\pi}^{\pi} \frac{\sin ^{2} \varphi}{\left[\sin ^{2} \varphi+(\lambda-\cos \varphi)^{2}\right]^{2}} d \varphi
$$

where $e_{0}(\lambda)=E_{0}(\lambda) / L$ and $s(\lambda)=S(\lambda) / L$. Both of the above integrals are divergent when $\lambda=1$. Their singular behavior can be analyzed in the vicinity of critical point $\lambda_{c}=1$ with the asymptotic behavior described by $-\partial^{2} e_{0}(\lambda) / \partial \lambda^{2}=-0.31187 \ln \left|\lambda-\lambda_{c}\right|+$ const and $\ln s(\lambda)=-\ln \left|\lambda-\lambda_{c}\right|+$ const, respectively.

To get further insight on how the two approaches work, we study both the scaling behaviors of FS and second derivative of GS energy. As shown in Fig. 1, we display the second derivatives of GS energy for different lattice sizes. Despite no real divergence for finite $L$, it is obvious that the curves exhibit marked anomalies with height of peak increasing with the lattice size. The parameter $\lambda_{m}$ labelling the position of peak approaches the critical point $\lambda_{c}=1$ in a way of $\lambda_{m}=1-$ const $^{-1.802}$. The value of peak diverges logarithmically with increasing lattice size as $-\partial^{2} e_{0}(\lambda, L) /\left.\partial \lambda^{2}\right|_{\lambda_{m}}=0.31132 \ln L+$ const. By proper scaling scheme, we can fit all the data of $F=\left[1-\exp \left(\partial^{2} e_{0} /\left.\partial \lambda^{2}\right|_{\lambda_{m}}-\partial^{2} e_{0} / \partial \lambda^{2}\right)\right]$ as a function of $L\left(\lambda-\lambda_{m}\right)$ for different $L$ into a single curve as displayed in the inset of Fig. 1. Correspondingly, the FS exhibits

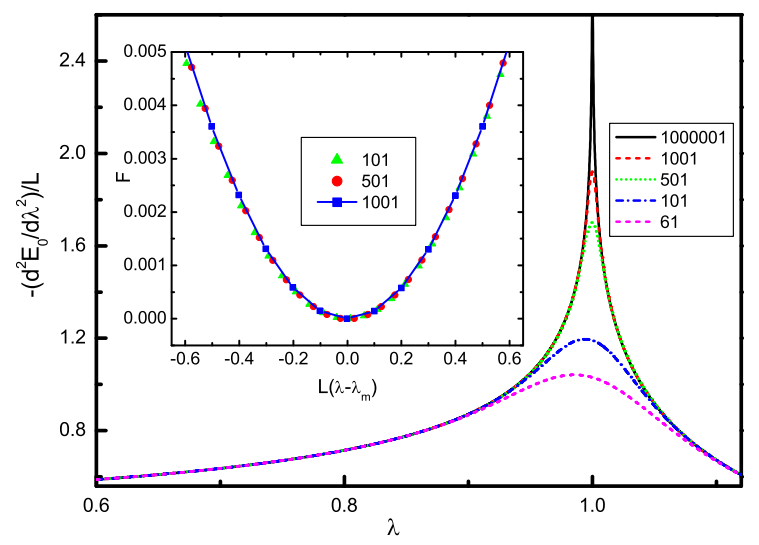

FIG. 1: (color online) The derivatives $\partial^{2} E_{0}(\lambda) / \partial \lambda^{2}$ as a function of $\lambda$.

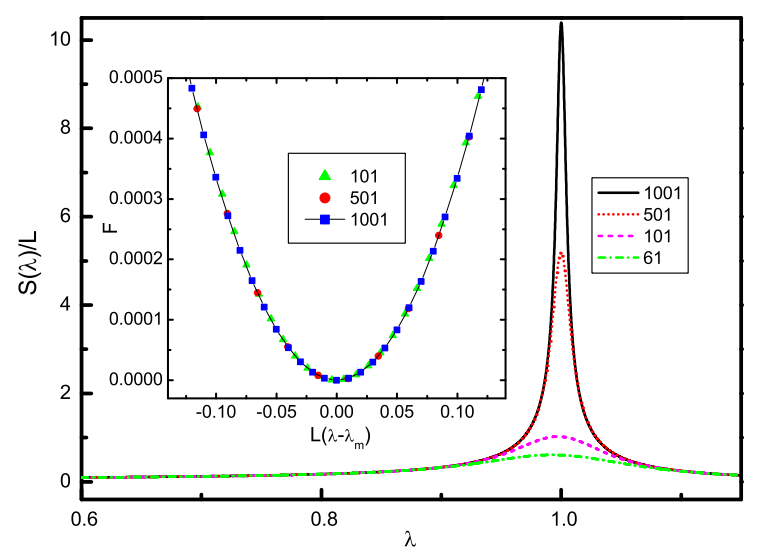

FIG. 2: (color online) The fidelity susceptibility as a function of $\lambda$.

similar scaling behaviors as shown in Fig. 2. Around the critical point, all the data for different $L$ collapse into a single curve of $F=\left[1-s(\lambda) / s\left(\lambda_{m}\right)\right]$ as a function of $L\left(\lambda-\lambda_{m}\right)$. A related quantity has been used in [13] to make scaling analysis for the quantum Ising model. It is clear that $\partial^{2} e_{0}(\lambda) / \partial \lambda^{2}$ and $s(\lambda)$ exhibit similar critical behavior around the critical point.

\section{IV. $X X Z$ SPIN CHAIN}

Having demonstrated the equivalent role of the two different approaches in identifying QPT on the transverse Ising model, we now use this procedure to understand the more elusive KT-type QPT for which, contrary to the previous expectation, the GS fidelity and the FS turn out to be insensitive to QPT in some cases, for example, in the spin $J_{1}-J_{2}$ model [14]. We now consider the $X X Z$ 


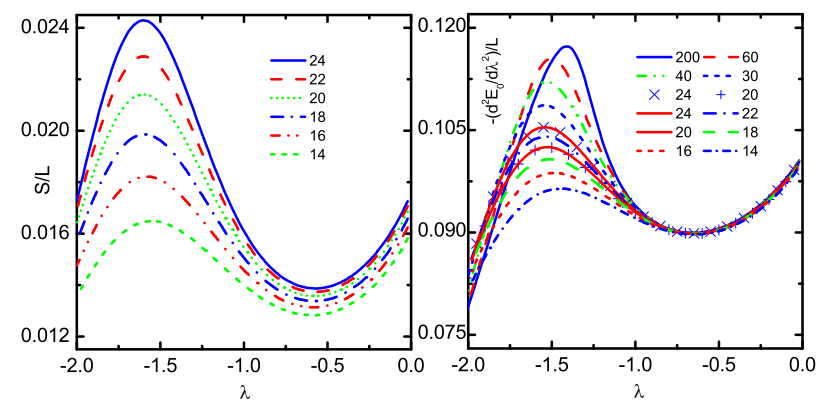

FIG. 3: (color online) The fidelity susceptibility and second order derivative of GS energy for $X X Z$ model as a function of $\lambda$. The data labelled by $\times$ and + are obtained by ED and they comply with the data by Bethe-ansatz method exactly.

model which provides a further test for the intrinsic relationship between the derivatives of GS energy and FS. The model is defined by

$$
H(\lambda)=-\frac{1}{2} \sum_{i=1}^{L}\left(\hat{\sigma}_{i}^{x} \hat{\sigma}_{i+1}^{x}+\hat{\sigma}_{i}^{y} \hat{\sigma}_{i+1}^{y}+\lambda \hat{\sigma}_{i}^{z} \hat{\sigma}_{i+1}^{z}\right)
$$

with $\lambda$ the exchange anisotropy parameter and it is related to $-H(-\lambda)$ by a unitary transformation. It is well known that the model is in a critical phase for $-1 \leq \lambda<1$, an antiferromagnetic phase at $\lambda<-1$, and ferromagnetic phase at $\lambda>1$. The QPT at the ferromagnetic isotropic point $\lambda=1$ is a $1 \mathrm{QPT}$ caused by the level crossing of GS. Here we shall focus on the QPT at the antiferromagnetic isotropic point $\lambda=-1$ which is known to be of KT type. In the whole parameter regime the model is integrable. The eigenstate of $H(\lambda)$ has the form of $|\Psi\rangle=\sum_{n_{1}<\cdots<n_{M}} a\left(n_{1}, \cdots, n_{M}\right) S_{n_{1}}^{-} \cdots S_{n_{M}}^{-}|F\rangle$ with the coefficients having the Bethe-ansatz form [20]

$a\left(n_{1}, \cdots, n_{M}\right)=\sum_{P} \exp \left[i \sum_{\alpha} k_{p_{\alpha}} n_{\alpha}+\frac{i}{2} \sum_{\alpha<\beta} \theta\left(k_{p_{\alpha}}, k_{p_{\beta}}\right)\right]$

where $P$ is any permutation of $\left(n_{1}, \cdots, n_{M}\right)$ and the phase $\theta(p, q)=2 \tan ^{-1}\left(\frac{\lambda \sin [(p-q) / 2]}{\cos [(p+q) / 2]-\lambda \cos [(p-q) / 2]}\right)$. The eigenenergies $E=-\frac{\lambda}{2} L+2 \sum_{i}\left(\lambda-\cos k_{i}\right)$ are determined by a set of quasi-momentum $p_{i}(i=1, \cdots, M)$ for $M$ down spins which are the solution of Bethe ansatz equations (BAEs) $N k_{i}=2 \pi I_{i}-\sum_{j=1}^{M} \theta\left(k_{i}, k_{j}\right)$, where $I_{i}$ are integer or half-odd integer.

By numerically solving the BAEs, we can get the GS state energy for different sizes of $L$. As shown in Fig.3, we calculate the 2nd derivative of GS energy by both Betheansatz and exact diagonaliztion (ED) methods and the FS by ED. Our numerical results show that there is no any singularity around the critical point $\lambda_{c}=-1$ for both the FS and 2nd order derivative of GS energy for different sizes. For the GS energy derivatives, this is true even in the thermodynamic limit where actually, as proven in 21], all the $n$th order derivatives of GS energy are continuous. Thus, in view of its connection to the derivatives of GS energy, we can understand why the GS fidelity failed to reproduce quantum critical behavior by the desired singularities in the critical point for the $\mathrm{KT}$ transition. It is also natural to explain why the finite size scaling of FS has a clear distinction between noncritical and critical phases because the GS energies are known to fulfil quite different finite size scaling in critical or noncritical phase [22, 23]. The GS energy in the critical regime fulfills the finite-size correction $E_{0}(\lambda, L) / L=e_{0}(\lambda)-\pi v(\lambda) c / 6 L^{2}+o\left(L^{-2}\right)$ with $c=1$ being the conformal anomaly number and $v=\pi \sin \gamma / \gamma$ the "sound velocity" for the model 22]. Here $\gamma$ is defined by $\lambda=-\cos \gamma$. On the other hand, the conformal invariance is broken for $\lambda<-1$ and the GS energy fulfills a different finite size scaling [23]. Bearing in mind that the equivalent role of FS and derivative of GS energy, we expect that the FS exhibits similar scaling behavior in the critical regime as its counterpart of derivative of GS energy does and there is a deviation from the scaling behavior when $\lambda<-1$. Indeed, the FS has been shown to scale differently in critical and noncritical phases based on the results by ED method [17]. This feature can be used as an indicator of KT QPT occurring in this model. Before ending the discussion, we would like to give a remark to the problem whether the fidelity approach is suitable to detect the KT transition? As no rigorous result of the FS even for the exactly solvable model is available in the thermodynamic limit, therefore this question is still in a doubtful status [15, 18] and remains an open problem.

\section{CONCLUSIONS}

We have shown the intrinsic relationship of the fidelity and derivatives of GS energy and revealed their equivalent role in identifying the QPTs. Within our framework, the singularities and scaling behavior of fidelity for various systems can be well understood on general grounds. Through concrete examples, we display that divergence of the FS or derivatives of GS energy can be applied to identify the 2nd order QPT, whereas for the KT transition the criticality is not a sufficient condition to ensure divergence of the FS or derivatives of GS energy.

\section{Acknowledgments}

The authors are grateful to M. F. Yang for useful discussions by correspondence and the referee for bringing our attention to Ref. [18]. This work is supported by NSF of China under Grant No. 10574150, MOST grant 2006CB921300 and programs of CAS. 
[*] Electronic address: schen@aphy.iphy.ac.cn

[1] S. Sachdev, Quantum Phase Transitions (Cambridge University Press, Cambridge, England, 1999).

[2] A. Osterloh, Luigi Amico, G. Falci and Rosario Fazio, Nature (London) 416, 608 (2002); T. J. Osborne and M. A. Nielsen, Phys. Rev. A 66, 032110 (2002).

[3] G. Vidal, I. Latorre, E. Rico, and A. Kitaev, Phys. Rev. Lett. 90, 227902 (2003).

[4] L. A. Wu, M. S. Sarandy, and D. A. Lidar, Phys. Rev. Lett. 93, 250404 (2004).

[5] S. J. Gu, S. S. Deng, Y. Q. Li and H. Q. Lin, Phys. Rev. Lett. 93, 086402 (2004).

[6] L. Amico, R. Fazio, A. Osterloh, V. Vedral, arXiv:quant-ph/0703044 [Rev. Mod. Phys. to appear].

[7] H. T. Quan, Z. Song, X. F. Liu, P. Zanardi, and C. P. Sun, Phys. Rev. Lett. 96, 140604 (2006).

[8] P. Zanardi and N. Paunkovic, Phys. Rev. E 74, 031123 (2006).

[9] P. Buonsante and A. Vezzani, Phys. Rev. Lett. 98, 110601 (2007).

[10] P. Zanardi, M. Cozzini, and P. Giorda, J. Stat. Mech. 2, L02002 (2007); M. Cozzini, P. Giorda, and P. Zanardi, Phys. Rev. B, 75, 014439 (2007); M. Cozzini, R. Ionicioiu, and P. Zanardi, ibid. 76, 104420 (2007).

[11] W. L. You, Y. W. Li, and S. J. Gu, Phys. Rev. E 76, 022101 (2007); S. J. Gu, H. M. Kwok, W. Q. Ning, and H. Q. Lin, arXiv:0706.2495.

[12] H. Q. Zhou, J. P. Barjaktarevic, arXiv: cond-mat/0701608, H. Q. Zhou, arXiv:0704.2945.

[13] H. Q. Zhou, J. H. Zhao, B. Li, arXiv:0704.2940 H. Q.
Zhou, J. H. Zhao, H. L. Wang, B. Li, arXiv:0711.4651,

[14] S. Chen, L. Wang, S. J. Gu, and Y. Wang, Phys. Rev. E 76, 061108 (2007).

[15] M. F. Yang, Phys. Rev. B 76, 180403(R) (2007); Y.-C. Tzeng and M. F. Yang, Phys. Rev. A 77, 012311 (2008).

[16] S. L. Zhu, Phys. Rev. Lett. 96, 077206 (2006); A. Hamma, arXiv:quant-ph/0602091 A. C. M. Carollo and J. K. Pachos, Phys. Rev. Lett. 95, 157203 (2005).

[17] L. C. Venuti and P. Zanardi, Phys. Rev. Lett. 99, 095701 (2007). P. Zanardi, P. Giorda, and M. Cozzini, Phys. Rev. Lett. 99, 100603 (2007).

[18] After the submision of the present paper (arXiv: 0801.0020v1), there appears a paper (L. Campos Venuti, M. Cozzini, P. Buonsante, F. Massel, N. Bray-Ali, and P. Zanardi, arXiv: 0801.2473v1), where an expression of fidelity susceptibility including the third order contributions is given.

[19] E. Lieb, T. Schultz, and D. Mattis, Ann. Phys. 16, 407 (1961); P. Pfeuty, Ann. Phys. 57, 79 (1970).

[20] M. Takahashi, Thermodynamics of 1D Solvable Models (Cambridge University Press, Cambridge, 1999).

[21] C. N. Yang and C. P. Yang, Phys. Rev. 150, 321 (1966); J. D. Cloizeaux and M. Gaudin, J. Math. Phys. 7, 1387 (1966).

[22] C. J. Hamer, J. Phys. A 19, 3335 (1986); L. V. Avdeev and B. D. Dorfel, J. Phys. A 19, L13 (1986).

[23] F. C. Alcaraz and M. J. Martina, Phys. Rev. Lett. 63, 708 (1989); H. W. Blöte et al., Phys. Rev. Lett. 56 , 742 (1986); I. Affleck, ibid. 56, 746 (1986). 